\title{
Thermal Testing of Ablators in the NASA Johnson Space Center Radiant Heat Test Facility
}

\author{
Steven Del Papa* \\ NASA Johnson Space Center, Houston, TX 77058 \\ Jim Milhoan \\ Barrios Technology, Houston, TX, 77058 \\ Brian Remark, Ph.D. ${ }^{\ddagger}$ \\ NASA Johnson Space Center, Houston, TX 77058 \\ Leonard Suess, Ph.D. ${ }^{\S}$ \\ Jacobs Technology, Inc., Houston, TX 77058
}

\begin{abstract}
A spacecraft's thermal protection system (TPS) is required to survive the harsh environment experienced during reentry. Accurate thermal modeling of the TPS is required to since uncertainties in the thermal response result in higher design margins and an increase in mass. The Radiant Heat Test Facility (RHTF) located at the NASA Johnson Space Center (JSC) replicates the reentry temperatures and pressures on system level full scale TPS test models for the validation of thermal math models. Reusable TPS, i.e. tile or reinforced carbon-carbon (RCC), have been the primary materials tested in the past. However, current capsule designs for MPCV and commercial programs have required the use of an ablator TPS. The RHTF has successfully completed a pathfinder program on avcoat ablator material to demonstrate the feasibility of ablator testing. The test results and corresponding ablation analysis results are presented in this paper.
\end{abstract}

\section{Introduction}

The Radiant Heat Test Facility (RHTF) shown in Figure 1 was designed and constructed for the development and certification tests of the Orbiter TPS. It consists of two altitude chambers, R1 and R2 (Figure 2) that can operate over a dynamic range from 0.067 to $101.3-\mathrm{kPa}(0.5$ to 760 -torr $)$ and contain an array of graphite heater elements capable of raising the temperature of the surface of a test article up to $1704^{\circ} \mathrm{C}\left(3100^{\circ} \mathrm{F}\right)$. R2 is $2.33-\mathrm{m}(92-\mathrm{in})$ diameter and $2.33-\mathrm{m}(92-\mathrm{in})$ in length. It has a single flat graphite heater array with a heated area of $0.61 \mathrm{x} 0.61-\mathrm{m}$ (24x24-in) and can handle models that are flat or possess a slight curvature. R1 is the larger test chamber that is $3.05-\mathrm{m}(120$-in) in diameter and $5.49-\mathrm{m}(18-\mathrm{ft})$ in length. It can test models up to $1.83 \times 2.79-\mathrm{m}(72 \times 110-$ in) that or flat or with a large amount of curvature. $\mathrm{R} 1$ can control up to 22 independent heater zones to accurately apply a temperature gradient across the surface of the test article. This was used with great success during the certification of the Orbiter nosecap (Figure 3) ) $^{1,2}$. Triangular and trapezoidal graphite elements were used to closely match the nosecap surface contour and the elements were grouped into zones to apply the time dependent temperature as shown in Figure 4. The facility has a 5-MW substation to drive the heaters and a closed loop cooling circuit for facility cooling. The test chambers may be tested in air or inert nitrogen as the test requirements dictate.

For the initial ablator test a small 10.16-cm (4-in) diameter, 5.08-cm (2-in) thick avcoat puck was used to determine the facility compatibility with the outgassing products for both safety and susceptibility to arcing. The heaters run at a low voltage (typically $100-\mathrm{V}$ or less) so arcing is usually not an issue, but if contaminants or particulates are introduced then arcing may occur as conduction paths are generated. Special circuitry is designed into the system to suppress arcing as it occurs, but repeated arcs may negatively affect the test results. Safety was also a concern as one of the expected byproducts of the pyrolization process is benzene. Since it is unknown the exact amount of benzene

${ }^{*}$ Aerospace Engineer, NASA Johnson Space Center, Thermal Design Branch ES3, Houston, TX 77058

$\uparrow$ Senior Engineer, Barrios Technology, Houston, TX, 77058

* Aerospace Engineer, NASA Johnson Space Center, Thermal Design Branch ES3, Houston, TX 77058

$\S$ Aerospace Scientist, Jacobs Technology, Inc., Houston, TX, 77058 
production a residual gas analyzer (RGA) was utilized to sample to chamber environment and calculate the maximum amount of benzene produced using overly conservative assumptions.

\section{Test Article and Test Setup}

The two pathfinder tests consisted of avcoat pucks with in depth type-R and type-K thermocouples (Figure 5). These were initially intended for arc jet testing and were initially bonded to an insulator on the backside. These were removed prior to testing in the radiant facility. The test article is surrounded with graphite felt and insulated on the back with LI-900 blocks to provide an adiabatic boundary for the sidewalls and backside.

The ablator model was tested in the R1 chamber with graphite felt as the surrounding insulating close-out (Figure 6 and Figure 7). The enclosed volume was approximately $133.4-\mathrm{cm}$ (52.5-in) long, 15.2-cm (6.0-in) wide, and 1.91$\mathrm{cm}(0.75$-in) tall with alumina tubes on end injecting a slow nitrogen purge. The nitrogen purge was designed to pass over the test article to sweep away particulates and out gassing products in a controlled manner. This would prevent a buildup of the contaminants that could possibly reduce the heating onto the test article, but with a velocity low enough to result in a negligible cooling effect on the test article. The purge would also force the byproducts over a water cooled copper plate immediately downstream of the test article to condense and collect the byproducts for analysis after the test. A wide angle broad spectrum total radiometer was installed upstream next to the test article to measure the incident radiant heat flux. A slight nitrogen purge was applied to interior ellipsoidal cavity of the sensor to prevent contamination that would cause any attenuation of the reading. Additional test environment data was obtained from a type $\mathrm{C}$ thermocouple embedded in the graphite susceptor plate.

The test setup as described above is installed on a lift system that vertically traverses from what is called the "home" position to the "heater" position. In the heater position all the radiant energy is contained within the cavity defined by the insulating closeout. This greatly improves the efficiency of the test as less power is required for obtaining the desired temperature. When the test article is at the home position the heater and the test article can radiate to the surrounding chamber environment. There is also a water cooled shutter that can be pneumatically inserted between the heater and the test article.

For the first avcoat ablator test (R1-123-15) the model was in the heater position when power was initiated to the heater. This setup results in a gradual ramp up of the heat flux to the test article as the elements and the susceptor plates heat up. The second test (R1-124-15) began with the test article in the home position with the shutter inserted. The power was applied to the heater for approximately 300 seconds while in this configuration. The shutter was then retracted and the test article raised into the heater position over a period of approximately 30 seconds. This test method is closer to a step impulse heating environment which is more similar to an arc jet test. For both tests a constant 30-KVA level of power was applied to heater and the test chamber was evacuated to 66-Pa ( 0.50 -torr) or lower for the entire duration of the test.

\section{Test Results and Analysis}

The test environments and in depth temperature responses for the two test runs are show in Figure 8 and Figure 9. For run 123 where the test article began in the heater position the ablator experienced a gradual heating the temperature of the susceptor plate and hence the incident heating rate gradually ramped up over the span of approximately 200 seconds. For run 124 power was applied to the heater for 300 seconds prior to the retraction of the shutter and the lifting of the test article to the heater position. The steady state temperature of the susceptor plate was approximately $1155^{\circ} \mathrm{C}\left(2111^{\circ} \mathrm{C}\right)$ prior to the shutter retraction due to radiant energy losses to the ambient chamber environment. The side of the shutter facing the heater is also highly reflective so once the shutter was retracted there was also a corresponding drop in the susceptor temperature down to $1086^{\circ} \mathrm{C}\left(1987^{\circ} \mathrm{F}\right)$ from the increase in losses to the ambient energy. Also, note the step increase of the radiometer reading once the shutter is retracted as well. As the test article is raised to the heater position the radiant losses decrease as the test article and the surrounding insulation temperature increases and the enclosed volume approaches equilibrium. The test method produces more of a step heating environment when compared to the previous run. The in depth temperatures as reported by the thermocouples are shown in Figure 9 with the temperature scale removed due to the data being ITAR restricted. Note the early rise and plateau of the temperature from run 124 (Figure 10). This is consistent with 
data obtained from arc jet testing as well as EFT-1 flight data which gives confidence that the radiant facility can be used to help understand the ablation physics.

Pre and post-test photos of avcoat model RHTF-3665 are shown in Figure 11. The overall appearance of the model differed from arc jet test model mainly in the overall structure and from the absence of melt and flow of the glass from the ablator. All of the cells appeared to have recessed into the honeycomb structure and the lack of a shear environment resulted in the honeycomb wall remaining intact. It is thought that there is minimal recession due to the typical ablation process as seen in arc jet testing where the surface is continually eroded through shear and the cell height reduction is avcoat shrinkage as the virgin material pyrolyzes and converts to char. What is striking is the height differences from cells to cell. Close inspection of the cross section shows that for cells with the most shrinkage the avcoat separated from the honeycomb walls thus being unconstrained. The cells that remained adhered to the cells walls developed internal lateral cracks as the pyrolysis zone propagated in depth.

A CHAR (Charring Ablator Response Code) avcoat model was built and validated based on EFT-1 avcoat material and arcjet test data. Due to the high manufacturing cost and long schedule, old Avcoat samples from the 2008 time frame were used in this test. This flavor of avcoat is not the same as was flown on EFT-1. One of the biggest differences from a thermal viewpoint is that the radiant samples had filled primer while the EFT-1 avcoat did not. The filled primer increases the thermal conductivity of both the virgin and charred avcoat material. Consequently, the EFT-1 avcoat model was run in CHAR using the outer thermocouple (TC1) as a boundary condition. Due to the configuration in the radiant facility CHAR was run in 1D with an adiabatic backwall. Figure 12 shows the CHAR analysis results compared to the test data. The EFT-1 avcoat model under predicts the thermocouple data and the EFT-1 avcoat using unfilled primer is most likely the reason. Another aspect to keep in mind is that the char conductivity variable in the ablation code is typically tuned to account for other modeling inaccuracies such as pyrolysis gas conductivity, heat of pyrolysis, etc. The tuning of that parameter is done with data obtained in arc jet testing. Since the radiant facility environment is fundamentally different from the convective arc jet environment it is possible that certain physical processes that is aggregated under the char conductivity variable behaves differently or is possibly nonexistent. One example would be the active oxidation of the avcoat during pyrolysis and of the char layer that would not be present in this pathfinder program due to the low chamber pressure combined with the applied nitrogen purge gas. With this in mind, testing ablators in a radiant environment could be used to determine these individual parameters. One proposal would be to fully char an ablator specimen that has in depth thermocouples and then subject it to various temperatures and pressures in an inert nitrogen environment to empirically derive the temperature and pressure dependent char conductivity.

Performing tests within the test chambers at the RHTF requires careful planning of any potential byproducts due to test article outgassing. Modifications to the normal operation configuration were performed to allow for ablative material to be tested within the RHTF test chambers. Benzene $\left(\mathrm{C}_{6} \mathrm{H}_{6}\right)$ is predicted to form from an ablative test article for temperatures approaching $2240 \mathrm{~F}(1500 \mathrm{~K})$. To help detect the outgassing by-products, a Residual Gas Analyzer (RGA) system has been incorporated. This system allows for the detection of benzene and other hydrocarbons.

The RGA system consists of a vacuum system (Pfieffer Vacuum HiPace-80 Pumping Station) - to transport the sampled gas, isolation valves to separate and control pressure, and a residual gas analyzer (Stanford Research Systems RGA300) to measure the spectrum of mass-to-charge ratios of a gas composition. A metering valve allows for precise pressure control in the range of $\sim 10^{-8}$ Torr to $10^{-4}$ Torr where the upper pressure limit is defined by the working parameters for the RGA. The RGA is a quadrupole mass spectrometer that is controlled via manufacturer software. The RGA outputs partial pressure for a range of species according to the ratio of mass to charge (M/Q).

A typical RGA data set (R1-124-15) is shown in Figure 13. Since benzene has a mass of 76 atomic mass units (a.m.u.), one can then zoom into the region consisting of 50-80 a.m.u. (as shown in Figure 14) to verify the presence of benzene in the sampled environment. The RGA system samples the chamber throughout the entire test duration; therefore, one can determine the mass of benzene produced as a function of time as shown in Figure 15.

The chamber pressure is relatively constant over the heating cycle of the test article which greatly simplifies the total mass calculation. The results of the total mass of benzene is given in Figure 16. 


\section{Conclusion}

The RHTF located at JSC has implemented facility modifications and successfully demonstrated the capability of testing ablator materials in a safe and reliable manner. These pathfinder tests provided useful insight into the avcoat ablation mechanism absent the convective environment. An in depth thermal response in a carefully controlled environment also allowed for a good comparison of the analytical ablation models. Testing in the RHTF has specific benefits compared to arc jet testing

Future testing plans include going to higher temperatures and for longer durations to expand the experience base and to test larger models. There is also a desire to determine the conductivity of the char material itself. An ablator specimen that is instrumented with in depth thermocouples will be brought to an elevated temperature for a significant amount of time to allow the specimen to fully char. The specimen will then be tested in the RHTF at various temperatures and pressures. This will provide the conductivity of the char material as a function of temperature and pressure and result in a more accurate ablation model.

Longer term plans for the RHTF are to perform combined thermal/mechanical test where a full scale section of the flight like structure with TPS is subjected to a reentry temperature profile with a dynamic ambient reentry pressure profile while simultaneously being subjected to the flight mechanical load profile.

\section{References}

1 Sherborne, William D. and Milhoan, James D., "A Radiant Heating Test Facility for Space Shuttle Orbiter Thermal Protection System Certification," $11^{\text {th }}$ Space Simulation Conference, September 1980.

2 Suppanz, Murray J. and Grimaud, John E., "Space Shuttle Orbiter Nose Cap and Wing Leading Edge Certification Test Program," $11^{\text {th }}$ Space Simulation Conference, September 1980.

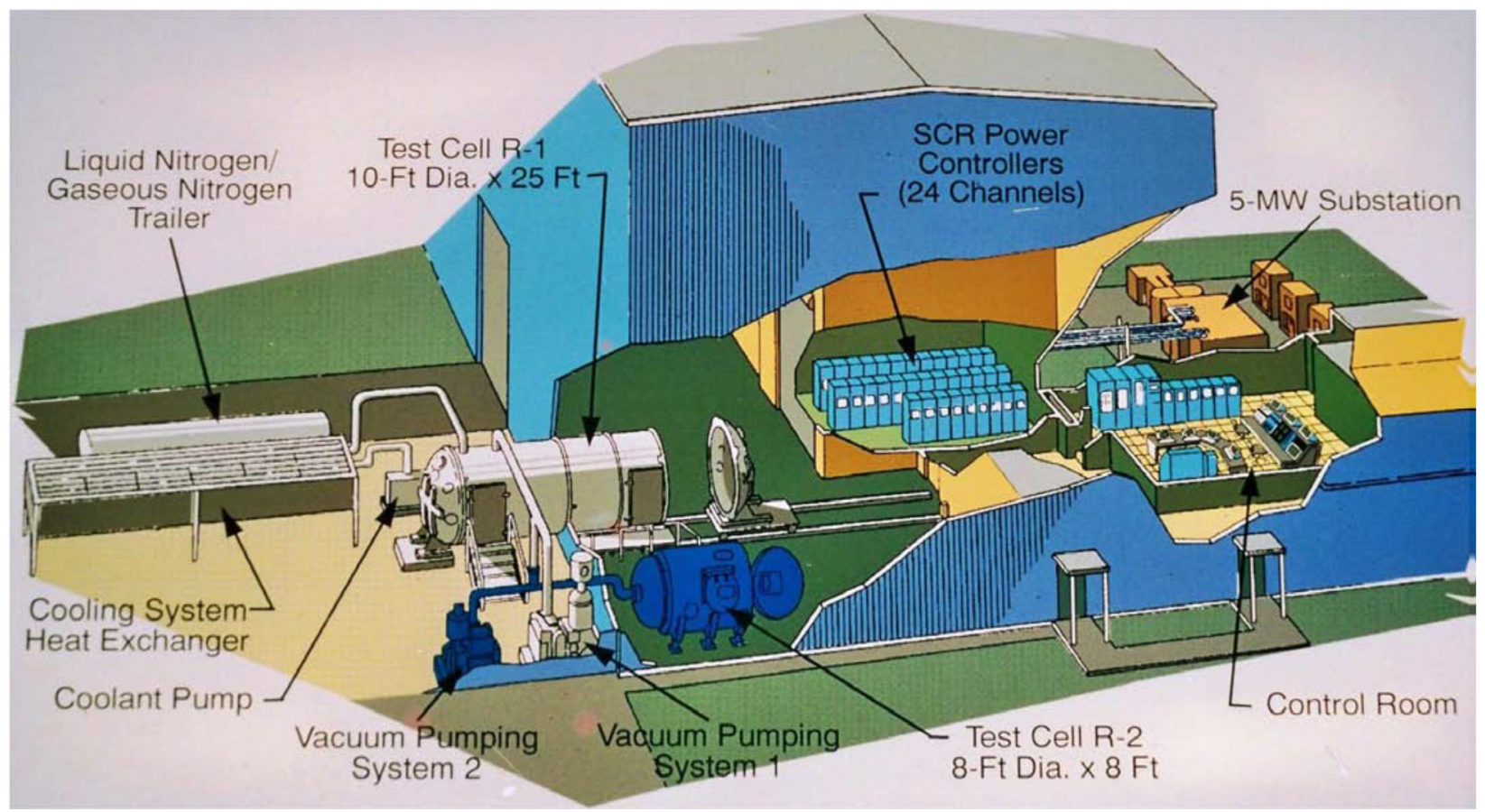

Figure 1: RHTF facility layout 

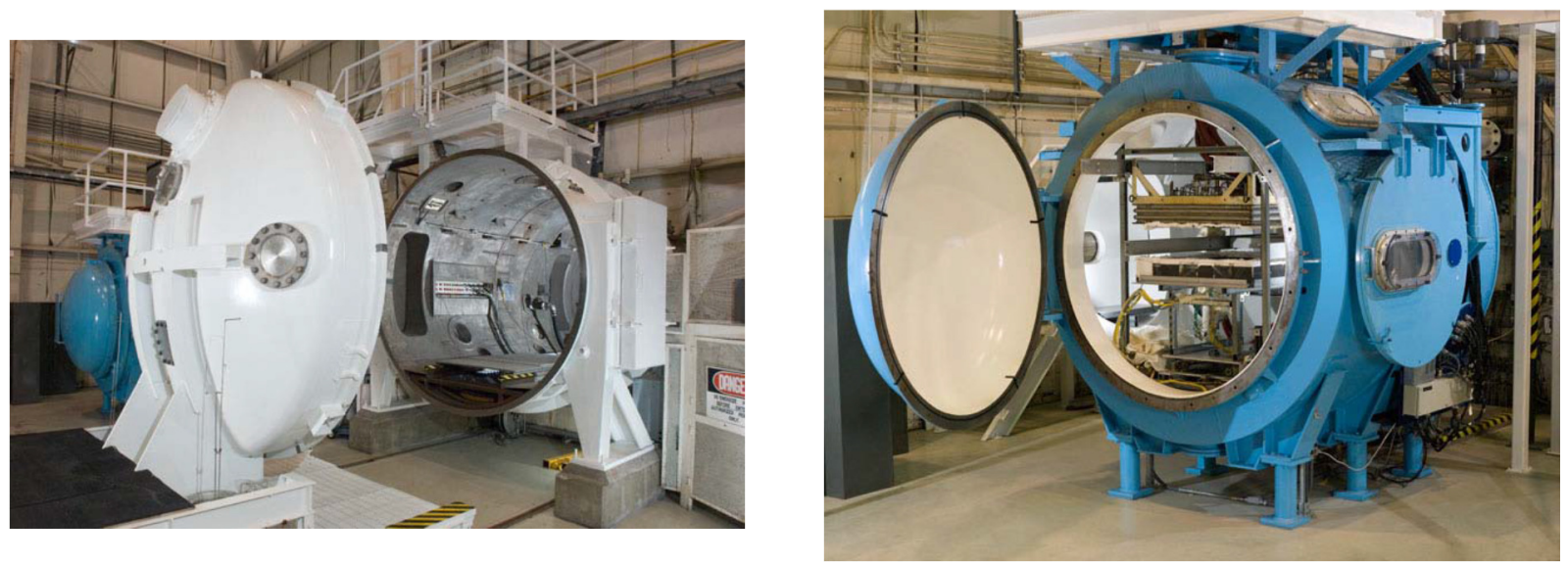

Figure 2: Radiant heat test chamber 1, R1 (left) and test chamber 2, R2 (right)

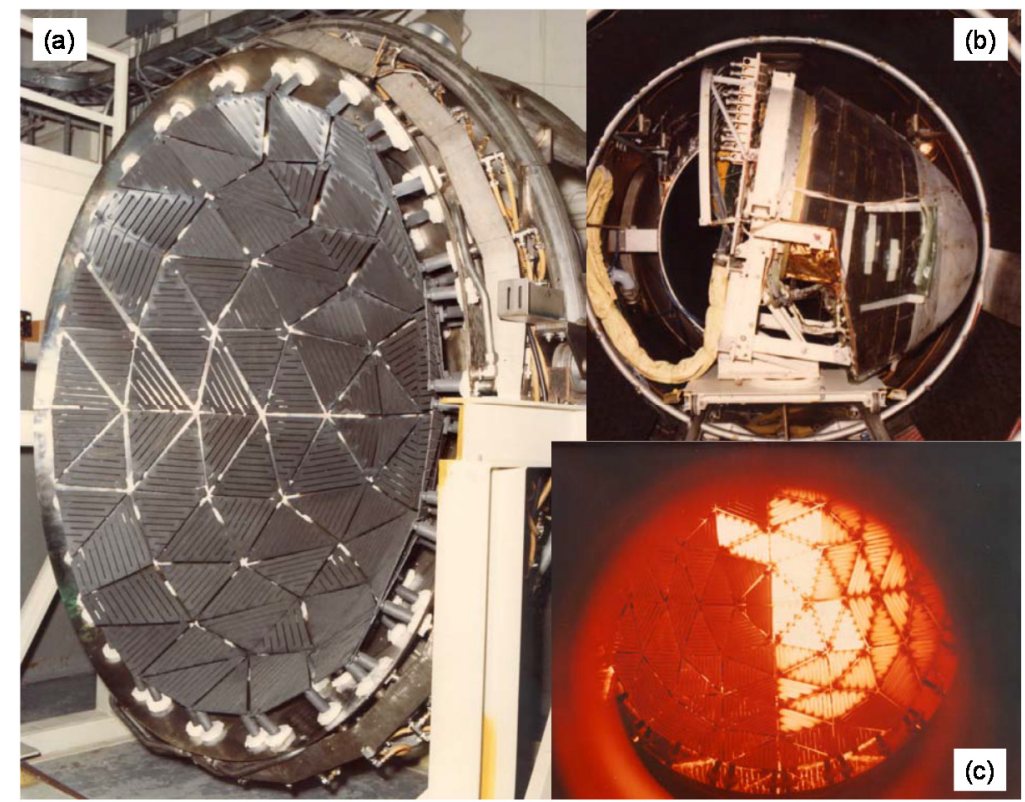

Figure 3: (a) Graphite heater array that approximates the exterior surface contour of the Orbiter nose cap; (b) Full scale Orbiter nosecap test article; (c) Graphite heater array in operation showing independently controlled heating zones

(a)

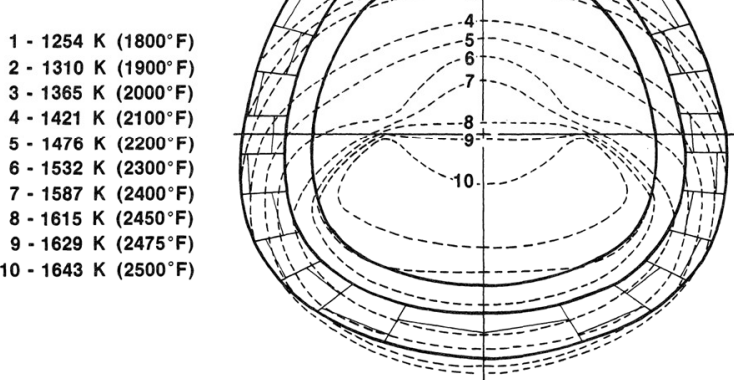

Figure 4: (a) Typical isotherms of the Orbiter nosecap; (b) Temperature profile of individual zones (b)

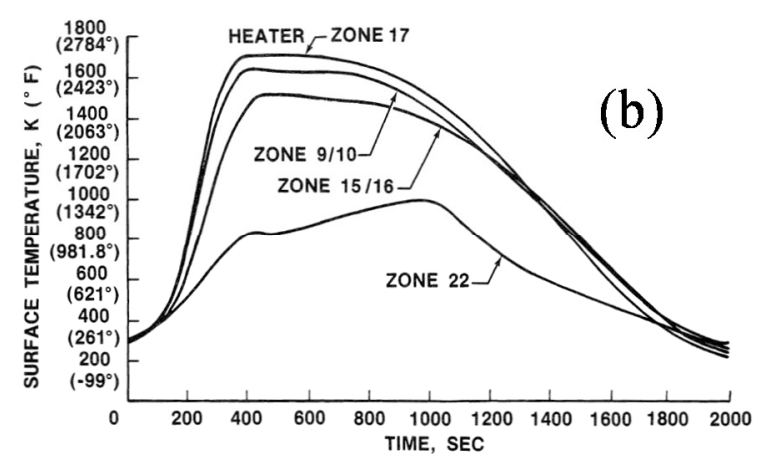




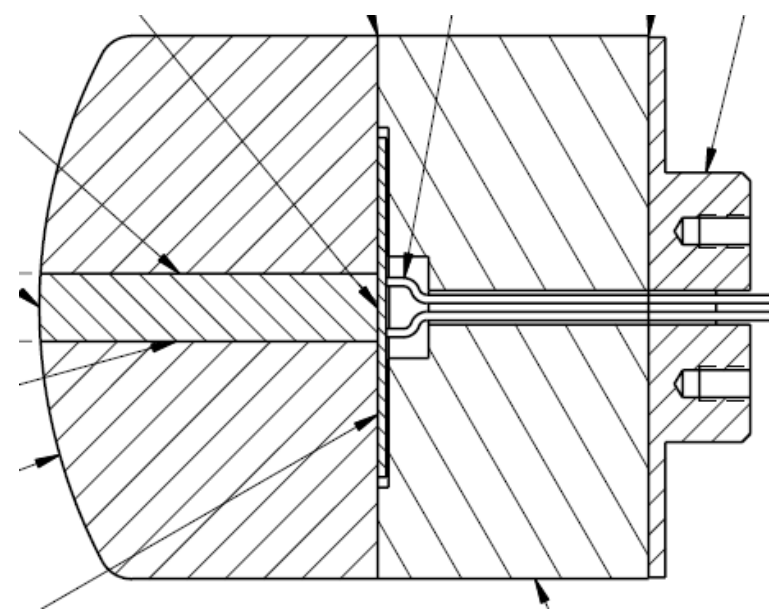

\begin{tabular}{|c|c|c|c|}
\cline { 4 - 4 } \multicolumn{2}{c|}{} & RHTF-3665 & RHTF-3670 \\
TC ID & TC Type & CT-43B-001 & CT-44A-002 \\
\hline TC01 & R & 0.205 & 0.303 \\
\hline TC02 & R & 0.248 & 0.398 \\
\hline TC03 & $\mathrm{K}$ & 0.299 & 0.496 \\
\hline TC04 & $\mathrm{K}$ & 0.508 & 0.591 \\
\hline TC05 & $\mathrm{K}$ & 1.008 & 0.804 \\
\hline
\end{tabular}

Figure 5: Pathfinder avcoat test articles (left) and thermocouple depths (inches) as measured by $\mathrm{x}$-ray (right)
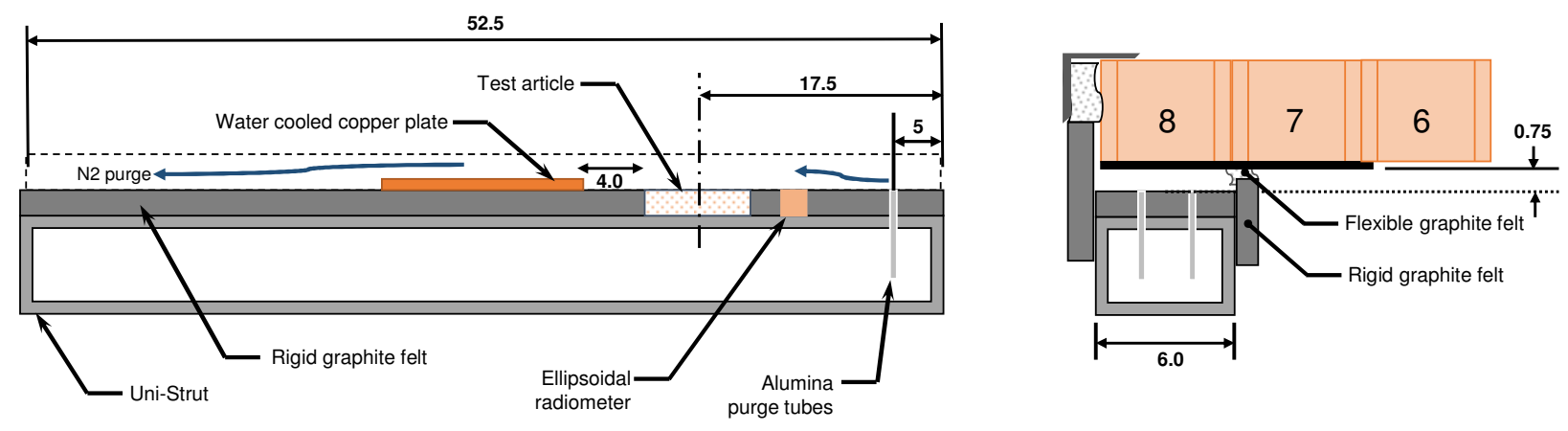

Figure 6: Test setup schematic 


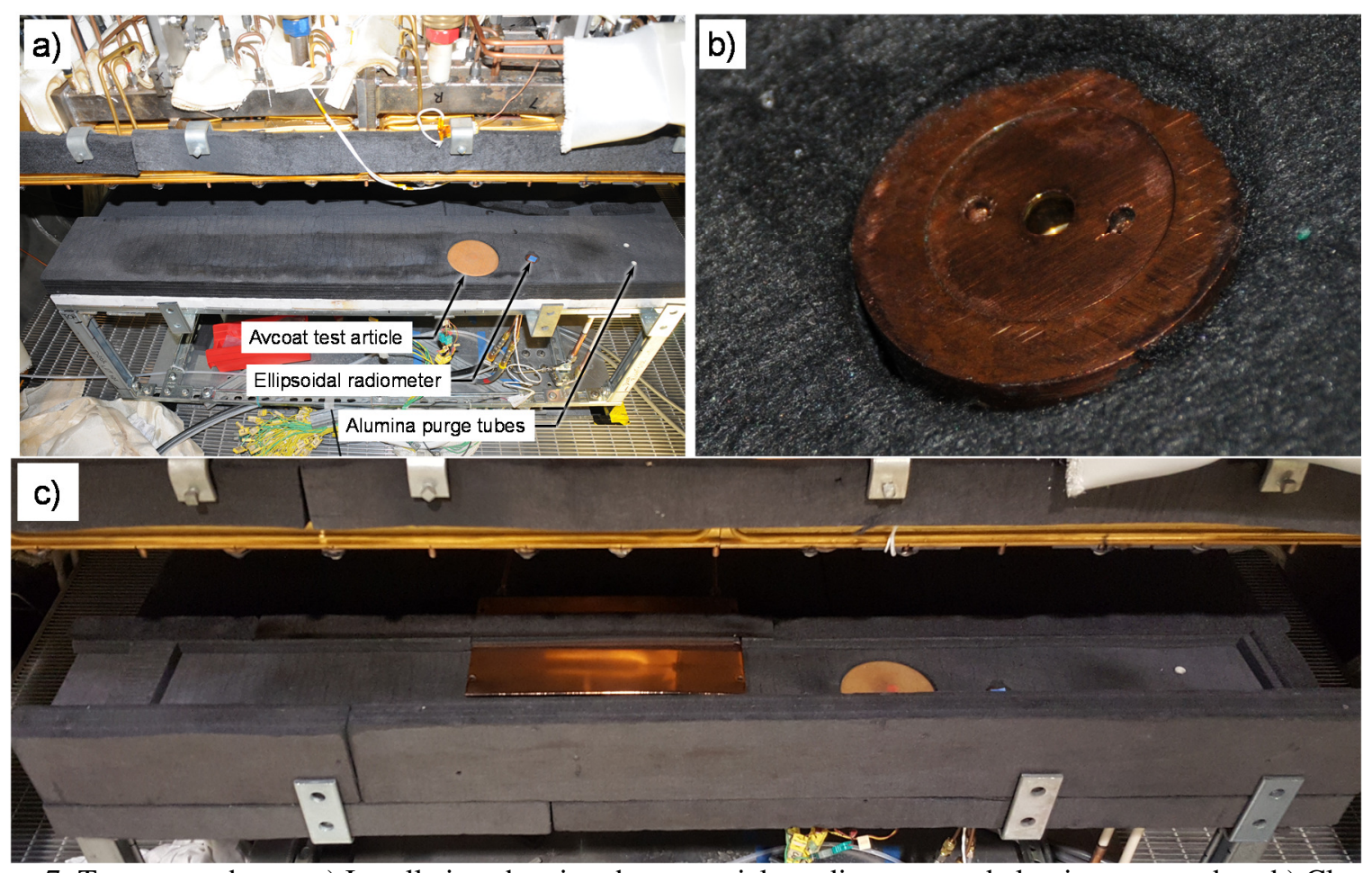

Figure 7: Test setup photos; a) Installation showing the test article, radiometer, and alumina purge tubes; b) Close up of the ellipsoidal total radiometer; c) Final test setup showing water cooled deposition panel and sidewall rigid graphite felt
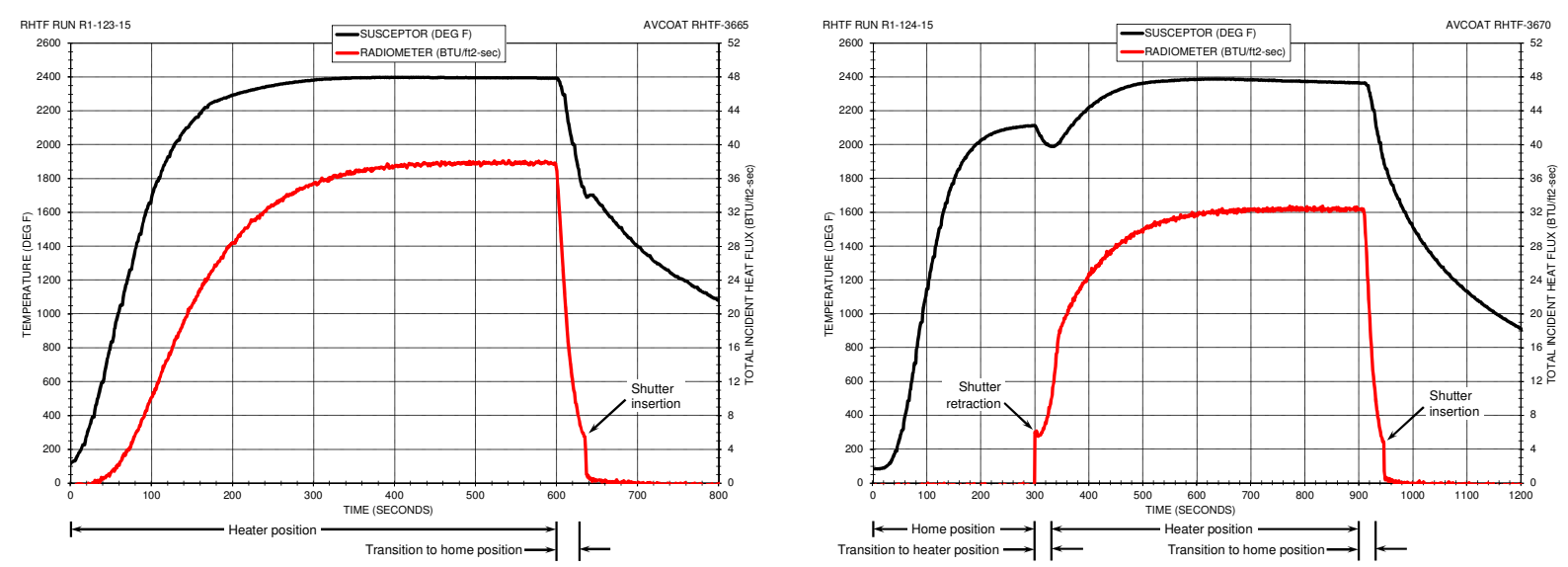

Figure 8: Sequencing and test environment for RHTF run R1-123-15 (left) and R1-124-15 (right) 

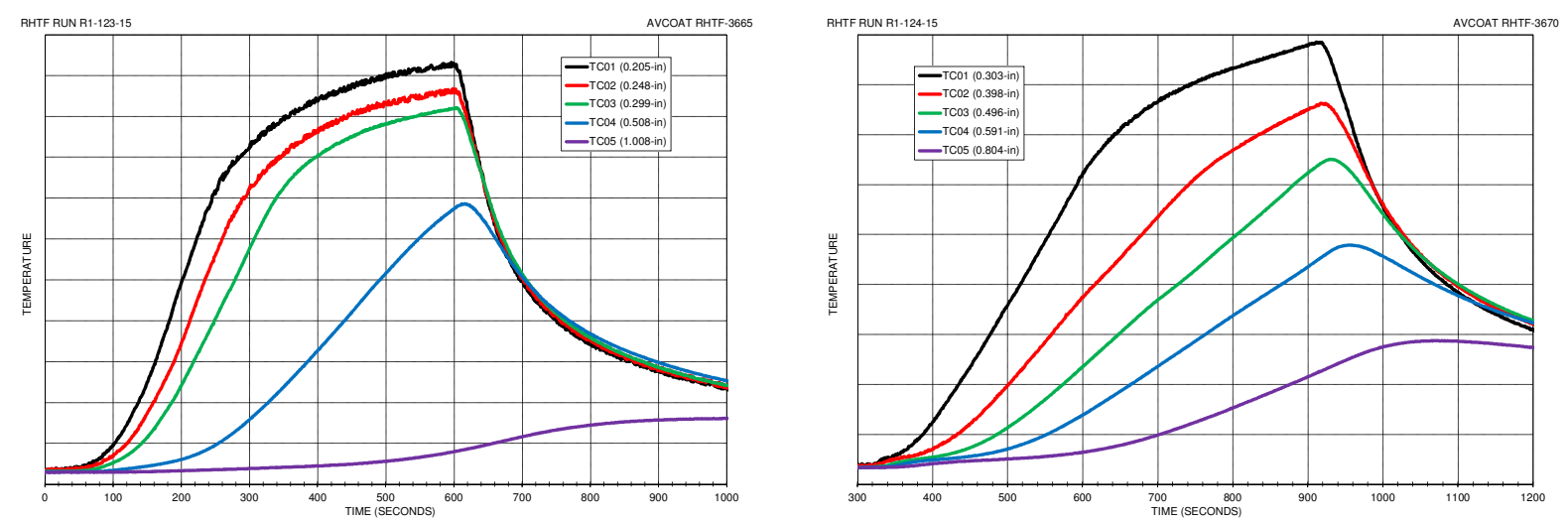

Figure 9: Thermocouple response for RHTF run R1-123-15 model RHTF-3665 (left) and RHTF run R1-124-15 model RHTF-3670 (right)

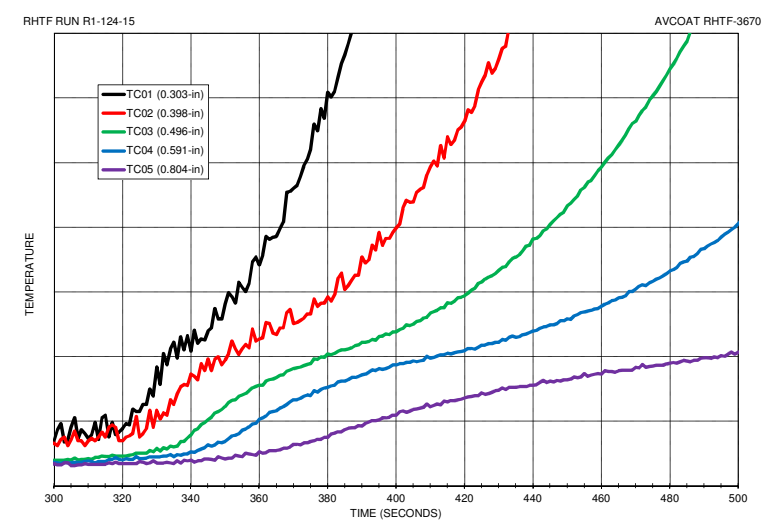

Figure 10: RHTF run R1-124-15 in-depth temperature plateau 

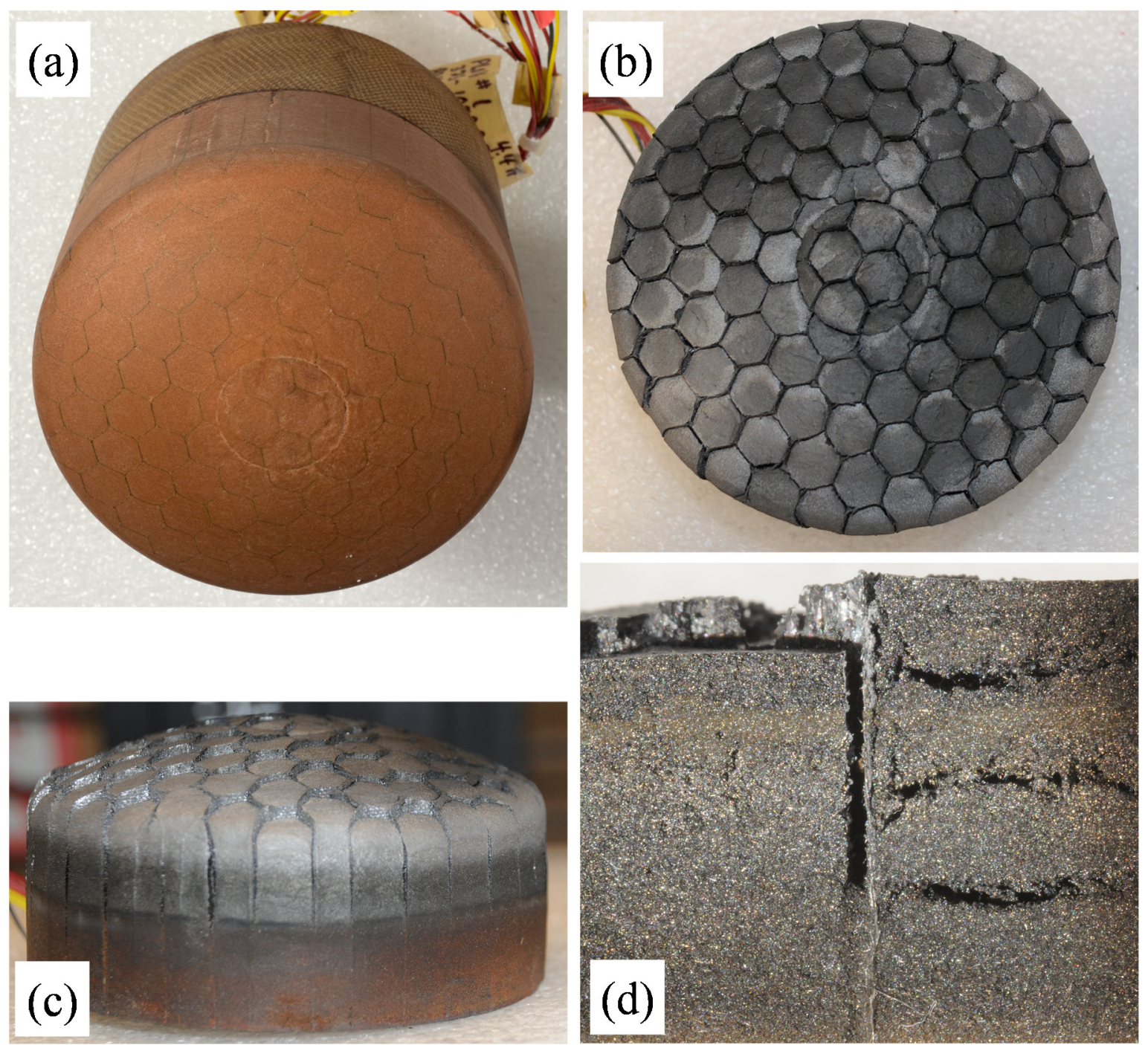

Figure 11: Photos of model RHTF-3665, a) pre-test oblique view, b) post-test normal view, c) post-test side view, d) post-test cross section close-up
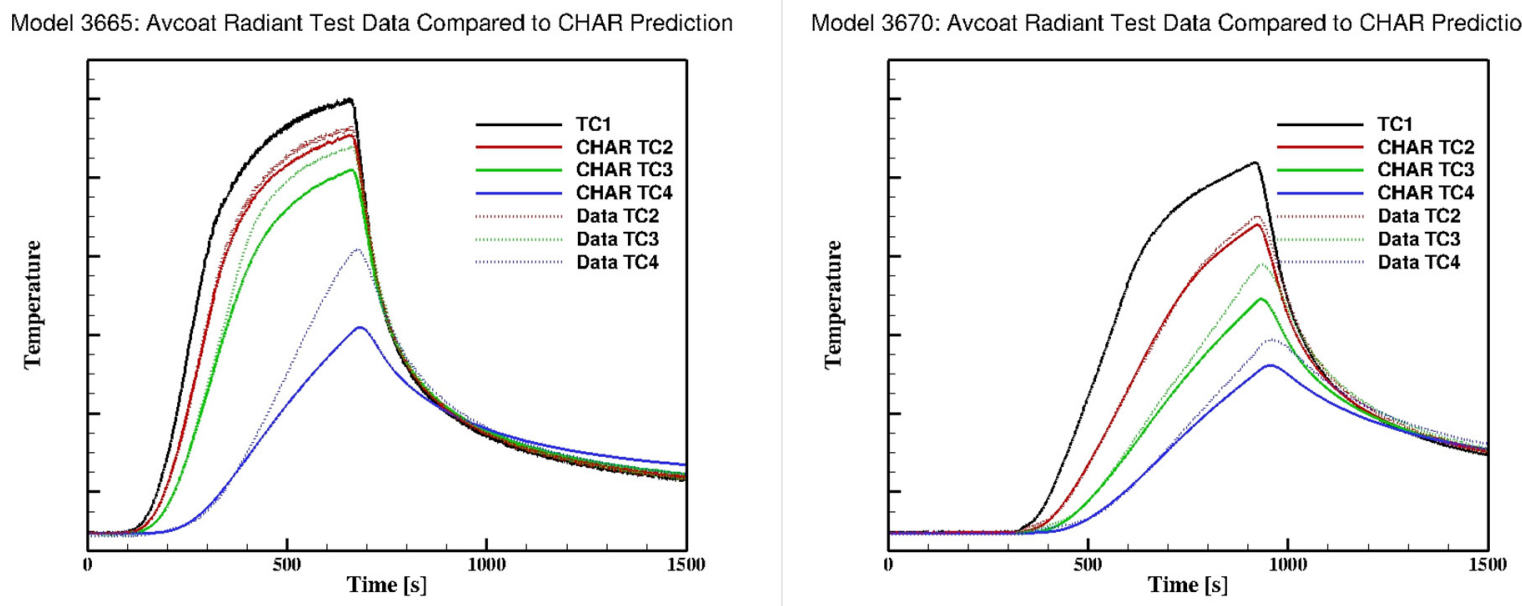

Figure 12: CHAR analysis compared to test data of RHTF run R1-123-15 model RHTF-3665 (left) and RHTF run R112415 model RHTF-3670 (right) 


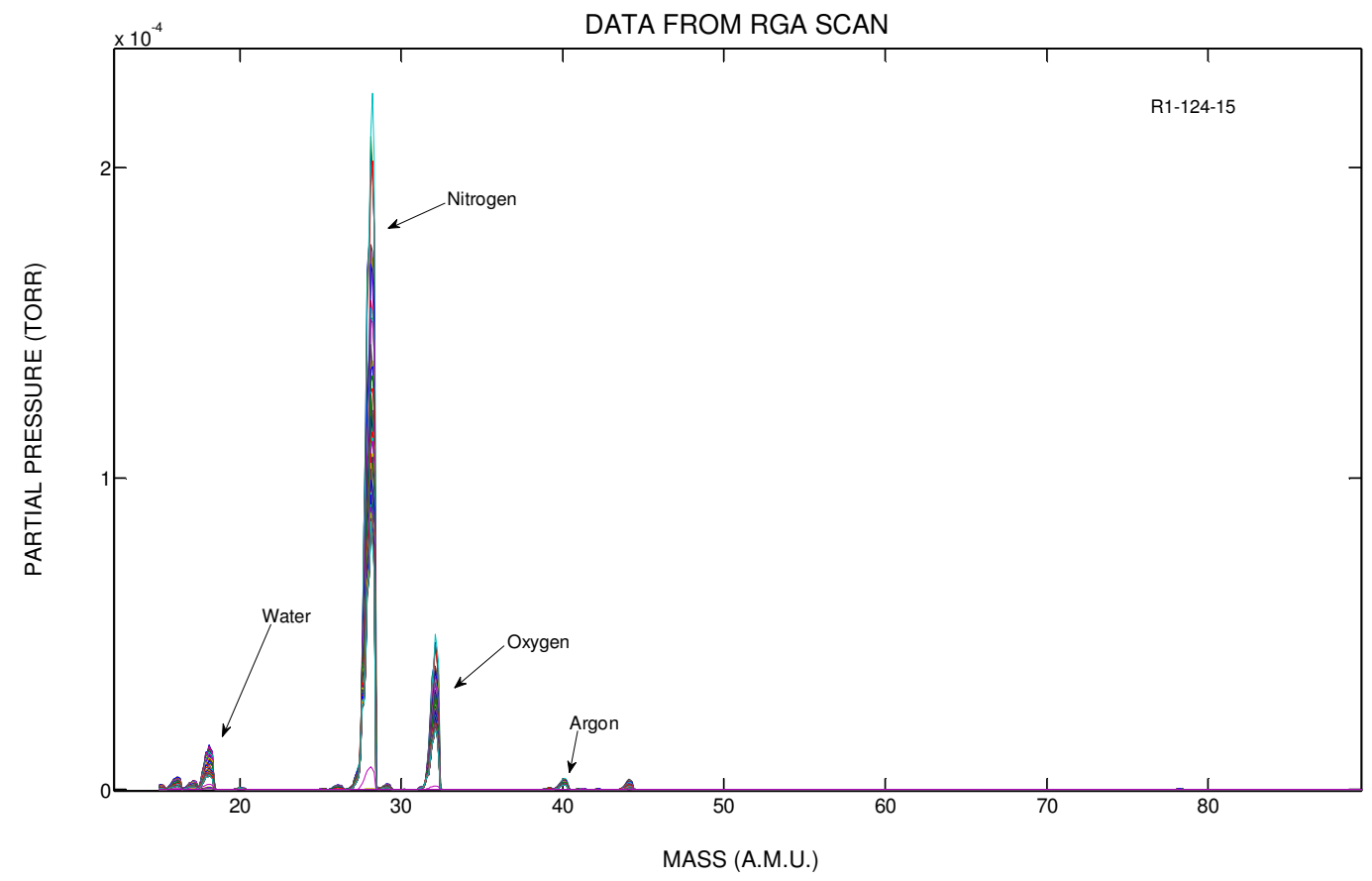

Figure 13: RGA results for run R1-124-15. M/Q as a function of time.

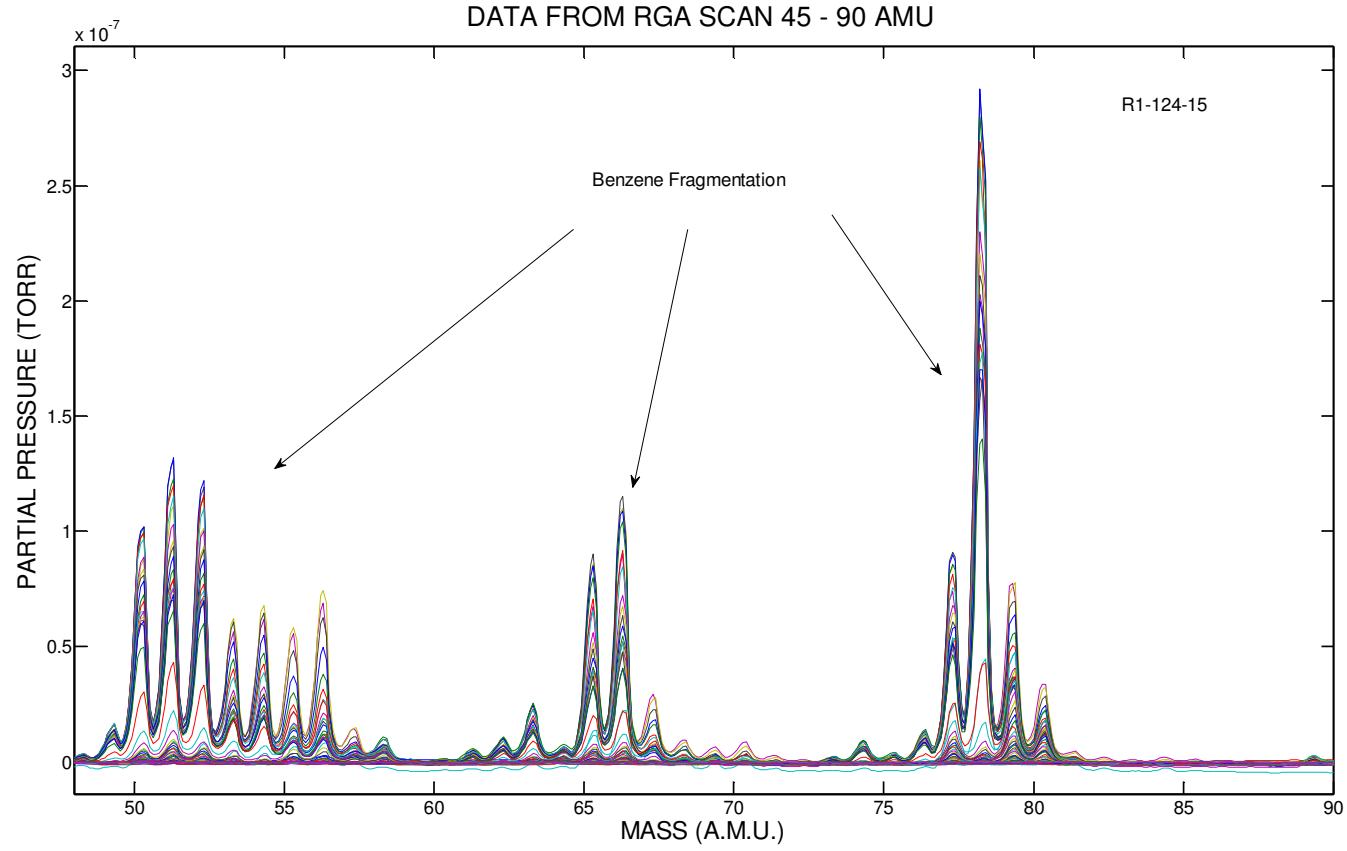

Figure 14: RGA results for run R1-124-15. M/Q as a function of time for M/Q: 45-90 AMU. 


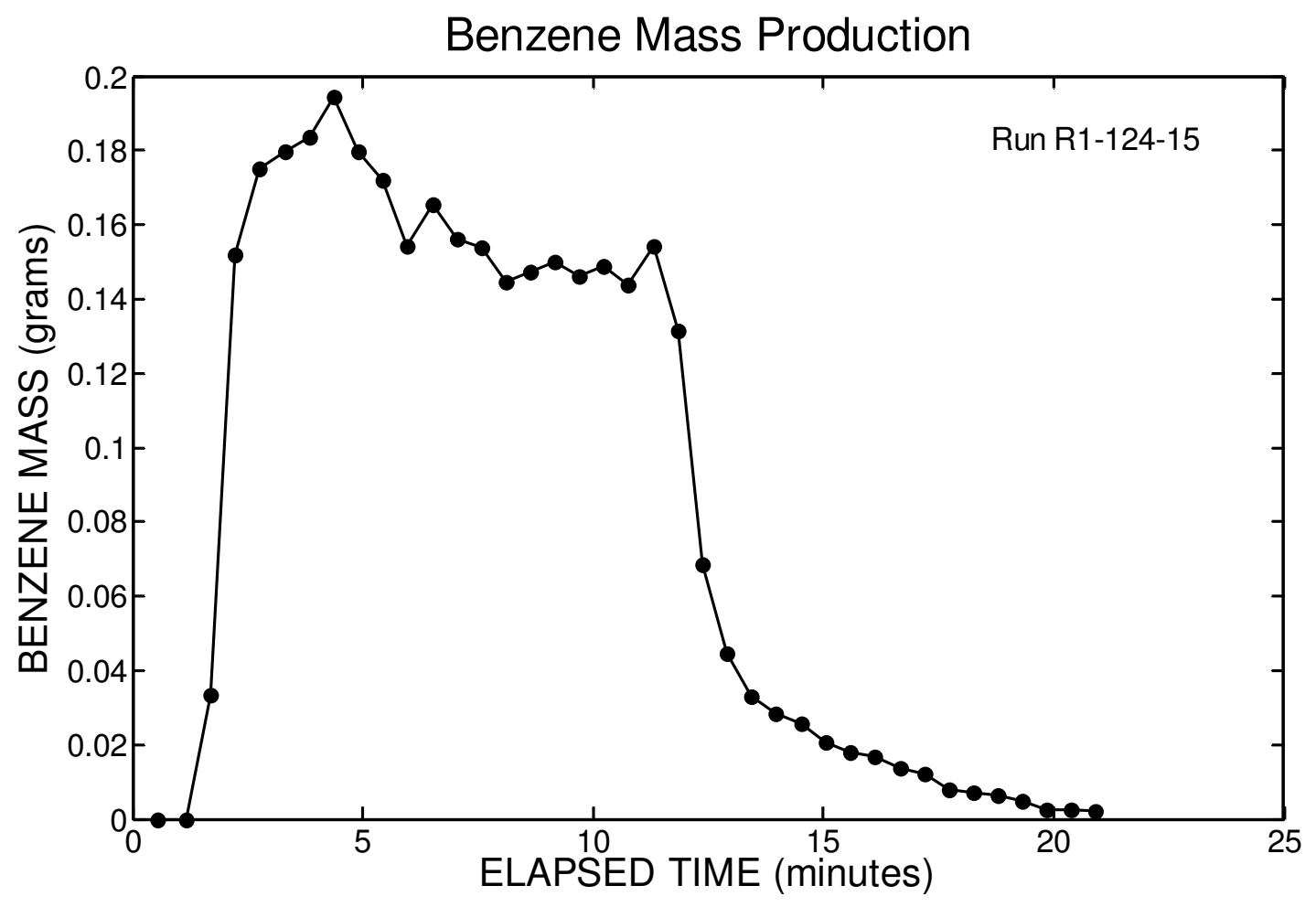

Figure 15: Benzene mass production as a function of elapsed time.

\begin{tabular}{|c|c|}
\hline Run \# & Total Benzene Produced (grams) \\
\hline \hline R1-123-15 & 0.89 \\
\hline R1-124-15 & 3.38 \\
\hline
\end{tabular}

Figure 16: Benzene mass production results. 